\title{
ELEMENTOS MINERAIS EM MADEIRAS DE EUCALIPTOS E ACÁCIA NEGRA E SUA INFLUÊNCIA NA INDÚSTRIA DE CELULOSE KRAFT BRANQUEADA
}

\section{MINERAL ELEMENTS IN WOODS OF EUCALYPTUS AND BLACK WATTLE AND ITS INFLUENCE IN A BLEACHED KRAFT PULP MILL}

\author{
André Freddo $^{1}$ Celso E. B. Foelkel ${ }^{2} \quad$ Sonia M. B. Frizzo ${ }^{3} \quad$ Maria C. M. Silva $^{3}$
}

\begin{abstract}
RESUMO
No ramo de papel e celulose os efluentes são um dos principais problemas e o consumo de água é crítico. Os circuitos internos estão sendo fechados e as fontes de contaminação precisam ser conhecidas. Uma das fontes de contaminação destes efluentes são os minerais da madeira, matériaprima neste tipo de indústria. Neste trabalho foi avaliada a quantidade de alumínio, cálcio, cobre, ferro, potássio, magnésio, manganês, sódio, níquel e silício em madeiras de cinco espécies florestais, plantadas na região de Guaíba, RS, compreendendo Acacia mearnsii, Eucalyptus dunnii, Eucalyptus globulus, Eucalyptus grandis, Eucalyptus saligna. As amostras de madeira foram tratadas com digestão ácida e com carbonização em mufla, solubilizando em ácido para serem analisadas por ICP (Inductively Coupled Plasm). Foi observado que a quantidade de elementos é considerável e os principais elementos encontrados foram $\mathrm{K}, \mathrm{Ca}, \mathrm{Na}, \mathrm{Al}, \mathrm{Mn}$, e Si. As espécies Eucalyptus grandis e Eucalyptus saligna mostraram melhor crescimento. Por outro lado, Eucalyptus globulus e Eucalyptus dunnii mostraram menor crescimento e maior teor de elementos. Pelo fato dessas espécies introduzirem maiores quantidades de elementos no processo de fabricação, devem conduzir a maiores problemas industriais. A Acacia mearnsii mostrou o menor nível para ferro, manganês e níquel, sendo uma boa opção para uso em branqueamento com oxigênio, ozônio e peróxido. $\mathrm{O}$ silício observado estava em baixa quantidade, o que leva a afirmar que há, na prática industrial, muita contaminação da madeira explorada com solo já que se encontram altos valores de sílica nos circuitos de licores. A entrada dos elementos minerais estudados em uma fábrica de celulose via madeira varia de 4 a $6 \mathrm{~kg}$ por tonelada de polpa não-branqueada produzida. Cuidados especiais devem ser tomados com as espécies com madeiras mais ricas em cinzas e elementos minerais e mais baixas eficiências nutricionais, como foi o caso de Eucalyptus dunnii e Eucalyptus globulus.
\end{abstract}

Palavras-chave: fechamento de circuitos, elementos minerais, madeira, metais, cátions, eucaliptos, acácia negra.

\section{ABSTRACT}

Effluents are one of the most important problems in a pulp mill regarding to environmental subjects. With the purpose to reduce them, the mills are closing the internal cycles and reducing the

1. Engenheiro Florestal, M.Sc. pelo Programa de Pós-Graduação em Enganharia Florestal. Centro de Ciências Rurais. Universidade Federal de Santa Maria. CEP: 97105-900. Santa Maria. RS.

2. Engenheiro Agrônomo, Dr., Professor Visitante do Departamento de Ciências Florestais. Centro de Ciências Rurais. Universidade Federal de Santa Maria. CEP: 97105-900. Santa Maria. RS.

3. Engenheira Florestal, M.Sc., Professora do Departamento de Química. Centro de Ciências Naturais e Exatas. Universidade Federal de Santa Maria. CEP: 97105-900. Santa Maria. RS. 
water consumption. The wood, as the most significant source of non-process elements to the system, is responsible for some troubles to the industrial process. With the aim of evaluating their intake and to offer some informations for closing the loop, the contents of $\mathrm{Al}, \mathrm{Ca}, \mathrm{Cu}, \mathrm{Fe}, \mathrm{K}, \mathrm{Mg}, \mathrm{Mn}, \mathrm{Na}, \mathrm{Ni}$, and $\mathrm{Si}$ were analysed in Acacia mearnsii, Eucalyptus dunnii, Eucalyptus globulus globulus, Eucalyptus grandis and Eucalyptus saligna woods. Wood samples were prepared by oven burning and acidic digestion methods, following analysis by ICP (inductively coupled plasm). Using also the results from silvicultural evaluation of trees and stands, they were calculated which elements were carried out from the site in larger amounts $(\mathrm{K}, \mathrm{Ca}, \mathrm{Na}, \mathrm{Al}, \mathrm{Mn}$ and $\mathrm{Si}$ ) and the species which exported largest amount of these elements (Eucalyptus dunnii and Eucalyptus globulus). The species with lower growth were Eucalyptus globulus and Eucalyptus dunnii, that leads to a bigger elements input and more potential industrial troubles. It was observed a range of 3.8 (Eucalyptus grandis) up to 6 (Eucalyptus dunnii) $\mathrm{kg}$ of analised mineral elements introduced to the process per ton of umbleached pulp produced. The Acacia mearnsii showed the lower level for $\mathrm{Fe}, \mathrm{Mn}$ and $\mathrm{Ni}$, being useful for oxygen, ozone and peroxide bleaching. The silicon observed in woods was in low concentration although the high values of this element in industrial liquor cycle. This leads to state that there is some contamination with soil when harvesting and handling the wood. Special care must be taken with both high ash and high mineral elements species, such as Eucalyptus dunnii and Eucalyptus globulus.

Key words: closing cycle, mineral elements, wood, metals, cations, ions, eucalyptus, black wattle.

\section{INTRODUÇÃO}

Os elementos minerais têm importância no desenvolvimento dos vegetais e seu papel é bastante conhecido. Nas árvores, suas quantidades variam com a espécie, as disponibilidades do solo, as necessidades individuais e outros fatores de menor importância. Atuam fisiologicamente nas folhas, mas são encontrados em todos os órgãos e tecidos, uma vez que são absorvidos do solo e levados através do tronco até a copa, participando do metabolismo vegetal.

No processo kraft, após a madeira ter sido convertida a celulose, resultam compostos residuais nos licores de cozimento, os quais possuem tanto componentes químicos vitais ao processo e que precisam ser recuperados, como também compostos minerais que são introduzidos pela madeira ou pelos insumos e que podem vir a se constituir em contaminantes. Estes últimos interferem na produtividade industrial e, quando se acumulam no processo, são causadores de problemas como corrosões, incrustações e entupimentos e, portanto, reduzem a vida útil dos materiais. Parte destes contaminantes permanecem nos efluentes, nos resíduos sólidos e aéreos. Por razões ambientais, a indústria de celulose tende para o fechamento de seu circuito de águas. Com isso, as perdas desses contaminantes via efluentes líquidos será cada vez mais limitada e eles tenderão a se acumular no processo, intensificando os problemas anteriormente descritos (ALBERT, 1993, EDSTROM, 1994, GRACE et al. e 1977).

ALBERT (1993) cita que a toxicidade dos efluentes é menos influenciada pelo processo de polpação e mais por compostos da própria madeira. A nível de comercialização, o mercado tende a 
utilizar produtos que tenham um menor impacto ambiental na sua produção e até aceita pagar um pouco mais por eles.

Todo o corpo técnico voltado ao branqueamento procura procedimentos básicos para o fechamento dos circuitos (monitoramento e controle de metais de transição, melhoria na concentração e evaporação de licores, reciclagem de efluentes do branqueamento, processos alternativos aos atuais, fechamento do circuito de branqueamento, redução de efluentes) sob condições industriais através de desenvolvimento de novos processos e criação das ferramentas necessárias para projetar plantas em ciclo fechado. Este tipo de pesquisa visa resolver não somente interesses ambientais, mas também para fornecer maior eficiência econômica na produção da celulose kraft (GLEADOW et al., 1993).

Devido aos problemas que os elementos minerais podem causar no processo de produção, a sua quantificação torna-se bastante útil para fornecer subsídios a medidas preventivas, uma vez que uma grande quantidade destes elementos minerais adentra à fábrica com a madeira.

Foram avaliados os teores de alumínio $(\mathrm{Al})$, cálcio $(\mathrm{Ca})$, cobre $(\mathrm{Cu})$, ferro $(\mathrm{Fe})$, potássio $(\mathrm{K})$, magnésio $(\mathrm{Mg})$, manganês $(\mathrm{Mn})$, sódio $(\mathrm{Na})$, níquel $(\mathrm{Ni})$ e silício ( $\mathrm{Si}$ ) em madeiras de Acacia mearnsii, Eucalyptus dunnii, Eucalyptus globulus, Eucalyptus grandis e Eucalyptus saligna.

Baseado nestes valores, determinou-se qual(is) espécie(s) contém maior ou menor quantidade destes elementos, buscando seus efeitos silviculturalmente (exportação de nutrientes) e industrialmente (a quantidade destes que ingressa com a madeira na fabricação de celulose).

\section{REVISÃO DE LITERATURA}

\section{Elementos minerais e a planta fabril}

EDSTROM (1994), cita que o teor de inorgânicos na madeira varia grandemente em função do local e forma como a espécie se desenvolve, fatores estes que devem ser considerados na instalação de uma indústria.

Os elementos que entram no sistema podem ser classificados em processuais e nãoprocessuais. Os processuais são $\mathrm{Na}$ e $\mathrm{S}$. Entre os não-processuais estão $\mathrm{Ca}, \mathrm{Mg}, \mathrm{K}, \mathrm{Cl}, \mathrm{C}, \mathrm{P}, \mathrm{Mn}, \mathrm{Al}$, $\mathrm{Si}, \mathrm{Fe}$ e, em menores quantidades, $\mathrm{Zn}, \mathrm{Cr}, \mathrm{Pb}, \mathrm{Hg}$, Ni e $\mathrm{Cd}$, podendo ainda ocorrer outros elementos. Destes elementos não-processuais, aproximadamente $60 \%$ entram via madeira, $35 \%$ como forma de produtos químicos processuais ou suas impurezas e 5\% com a água de processo (ALBERT, 1996).

De acordo com ALBERT (1995), a presença de elementos minerais não-processuais pode causar vários problemas na industrialização da madeira à celulose. Outra fonte destes elementos é o desgaste de equipamentos metálicos.

\section{Cozimento e licores}

Devido às exigências das indústrias para reduzir significativamente as emissões à atmosfera, 
diminuir o lançamento de efluentes e o consumo de água de processo, há uma tendência de fechamento do ciclo de produção (EDSTROM, 1994). Também informa que 1/3 das fábricas de celulose kraft branqueada produzem polpa sem utilizar cloro elementar como agente branqueador e também cita que o termo 'fechado' deve ser entendido como um mínimo de efluentes lançados nos cursos d'água.

GRACE et al. (1977), analisando a composição de inorgânicos em licores negros alcalinos, encontraram, dentre outros, $\mathrm{Na}_{2} \mathrm{SO}_{4}, \mathrm{Na}_{2} \mathrm{~S}, \mathrm{Na}_{2} \mathrm{~S}_{2} \mathrm{O}_{3}, \mathrm{NaCl}$, sódio total e potássio. A composição média de inorgânicos nestes licores está apresentada na Tabela 1.

TABELA 1: Quantidades de substâncias inorgânicas em licores negros alcalinos (base seca).

\begin{tabular}{c|cccccc}
\hline Substância & Teor & Substância & Teor & Substância & Teor \\
\hline $\mathrm{Na}_{2} \mathrm{CO}_{3}$ & $8,7 \%$ & $\mathrm{~S}$ (calc.) & $60,7 \%$ & $\mathrm{Fe}$ & $91 \mathrm{ppm}$ \\
$\mathrm{Na}_{2} \mathrm{SO}_{4}$ & $3,2 \%$ & $\mathrm{Ca}$ (abs. at.) & $360 \mathrm{ppm}$ & $\mathrm{Mn}$ & $96 \mathrm{ppm}$ \\
Alcali ativo & $5,95 \%$ & $\mathrm{Ca}$ (emissão) & $450 \mathrm{ppm}$ & $\mathrm{B}$ & $202 \mathrm{ppm}$ \\
$\mathrm{Na}$ & $18,7 \%$ & $\mathrm{Mg}$ & $184 \mathrm{ppm}$ & $\mathrm{K}$ & $1,36 \%$ \\
$\mathrm{~S}$ cinza (med) & $62,1 \%$ & Sílica & $2440 \mathrm{ppm}$ & $\mathrm{Al}$ & $139 \mathrm{ppm}$ \\
\hline
\end{tabular}

Fonte: GRACE et al. (1977)

\section{Branqueamento}

FOELKEL \& ASSIS (1995) realizaram um estudo baseado na sensibilidade das substâncias usadas em branqueamento "Totally Chlorine Free" (TCF) à ação do Fe e Mn, indicando que estes elementos decompõem o peróxido de hidrogênio e influenciam na degradação dos carboidratos. Os elementos não-processuais causam prejuízos à produção e contaminação do produto final. Como sugestão para a eliminação destes elementos pode ser utilizado o processo de precipitação eletrostática nas chaminés da fábrica com subsequente lavagem das cinzas com separação ou não de compostos processuais $\left(\mathrm{Na}^{+}, \mathrm{SO}_{4}^{--}\right)$dos não-processuais $(\mathrm{Ca}, \mathrm{Mg}, \mathrm{K}$ e $\mathrm{Al})$, seguida de filtração de licores.

Segundo EDSTROM (1994), se no branqueamento é usado peróxido de hidrogênio, alguns elementos inorgânicos, como Mn, precisam ser removidos da polpa pois poderão decompor o peróxido. Para isso, pode-se usar um quelante (como ácido dietilenodiaminotetracético - EDTA) ou recuperar o Mn por filtração de licor verde. Já sílica e fósforo, elementos também prejudiciais no processo podem ser removidos a partir do forno de cal, via purga da lama de cal.

LINDBERG (1994) cita que para remover os metais, evitando que estes não sejam reusados no processo, uma maneira eficiente é a quelação (com EDTA ou ácido dietilenotriaminopentacético DTPA), em meio levemente ácido. Outra forma simples para removê-los consiste em uma lavagem ácida no branqueamento e posterior eliminação destes resíduos. Como desvantagem dessa lavagem há eliminação do magnésio, que tem efeito benéfico nos estágios com oxigênio e peróxido.

BRYANT \& EDUARDS (1994) compararam o fluxo de elementos minerais em três fábricas com processos kraft. Os autores apresentaram um balanço de metais para estas fábricas, que demonstra que a maior fonte de elementos minerais para este tipo de indústria é a madeira. Nas 
fábricas estudadas, a que utiliza cavacos com casca, $50 \%$ do Mn e $25 \%$ do Fe são retidos pela polpa e vão para o branqueamento, comparado a $30 \%(\mathrm{Mn})$ e $60 \%$ (Fe) das fábricas que utilizam cavacos sem casca.

DEVENYNS et al. (1995), estudaram o controle de metais em um branqueamento TCF e citam que os metais de transição decompõem o peróxido de hidrogênio em água e oxigênio devido à capacidade de trocar o seu estado de oxidação. Destes, os mais reativos são o ferro, manganês e cobre. $\mathrm{O}$ cobre e o ferro estão fortemente fixados à pasta em forma precipitada de hidróxido, sendo o Mn o mais nocivo, devendo por isso, ser eliminado. O procedimento mais correto para esta eliminação consiste numa lavagem ácida da pasta.

Um efeito interessante é que não existe um aumento proporcional de alvura no branqueamento com a retirada progressiva de manganês na lavagem ácida. Um outro artifício utilizado no branqueamento com peróxido é a introdução de magnésio para aumentar a relação $\mathrm{Mg} / \mathrm{Mn}$ e suprimir a degradação de peróxido pelo Mn. Para uma otimização, uma relação de 30:1 (Mg:Mn) deve ser observada. Porém, um excesso de magnésio pode levar a uma sobra de peróxido no branqueamento com menor eficiência no branqueamento (DEVENYNS et al., 1995).

De acordo com STRÖMQVIST (1994), os filtrados do branqueamento não precisam recircular no sistema de recuperação, podendo ser tratados separadamente. Desta forma, as alterações nas condições processuais antes do branqueamento não devem mudar. Nos processos ECF e TCF, os elementos não-processuais devem ser balanceados (sílica, alumínio, manganês e potássio). Isto requer filtração adicional no sistema de recuperação para evitar problemas operacionais. Além disso, a purga por filtração afetará negativamente o balanço de químicos processuais. O evaporador e o sistema de recuperação não estarão capacitados, em muitos casos, para a quantidade extra de carga devido à simples falta de capacidade disponível.

Foi estudado por LINDBERG (1994) a ação de alguns elementos minerais como ferro, manganês, bário, cálcio, alumínio e sílica no branqueamento. Uma filtração dos licores verde e branco é a primeira estratégia para o controle dos metais em uma fábrica de polpa kraft. Isto ocorre pois o manganês tem uma solubilidade dez vezes menor no licor verde comparado ao licor branco, o que permite manter o teor de manganês no licor branco muito baixo, permitindo o uso deste licor em branqueamento de polpa até mesmo no estágio com peróxido.

LINDBERG (1994) e ALBERT (1995) relatam que vários trabalhos já foram realizados para estudar o controle e remoção de metais no processo fabril. Porém, HOBBS \& ABBOT (1994) citam que a adição de alumínio no licor de branqueamento (na forma de nitrato de alumínio) na polpação mecânica de Eucalyptus regnans pode aumentar a alvura em 2-3\% (dois a três) ISO se a sequiência com peróxido de dois estágios (ácido/alcalino) for empregada, em comparação com branqueamento de estágio alcalino único. O mecanismo pelo qual ocorre tal incremento em alvura, possivelmente seja pela interação do alumínio adicionado com o manganês presente na polpa, o que leva a uma redução do consumo de peróxido. Entretanto, se a adição de alumínio ocorrer acima de uma determinada dose crítica, ocorre estabilização do licor de branqueamento.

A implicação do fechamento dos circuitos para o ambiente apresenta a vantagem de que os rios e outras águas não servirão como recipientes para descargas prejudiciais. Além disso, o aumento

Ciência Florestal, v.9, n.1, 1999 
da deslignificação com oxigênio antes do branqueamento tem, nesta etapa, diminuído a necessidade de químicos (EDSTROM, 1994).

\section{Sistema de recuperação}

VENTURA \& TURQUETI (1994), avaliaram o balanço de cloretos e potássio no sistema de recuperação de licor da Riocell S. A. durante a purga das cinzas por um determinado período de tempo. Encontraram uma perda de $96 \mathrm{~kg} /$ dia de cloretos e um acúmulo de $51 \mathrm{~kg} / \mathrm{dia}$ de potássio. Segundo os autores, os cloretos dificultam a redução da temperatura de fusão das cinzas da caldeira de recuperação, enquanto o K não interfere neste processo.

Conforme TRAN et al. (1990), estudando os íons cloretos e potássio na recuperação dos químicos do processo kraft concluem que do ponto de vista da caldeira de recuperação, o potássio não causa um problema significativo se o conteúdo de cloretos no licor negro não queimado é menor que $1,5, \mathrm{Cl}^{-} /\left(\mathrm{Na}^{+}+\mathrm{K}^{+}\right)$(base molar).

Em fábricas ECF, o alto conteúdo de cloretos nos efluentes provenientes do estágio de dióxido de cloro do branqueamento traz um problema adicional para a reciclagem. As tecnologias desenvolvidas nos últimos 30 anos permitiu diminuir a quantidade de água requerida para produzir uma tonelada de polpa de $200-250 \mathrm{~m}^{3}$ para $10 \mathrm{~m}^{3}$. Futuramente a quantidade a ser usada para o processo de fabricação será equiparável ao volume de água que chega junto com a madeira. Ainda será possível produzir, além da polpa, eletricidade para a planta e fertilizantes para as florestas (EDSTROM, 1994).

MOUDGIL \& PRAKASH (19_), estudando técnicas de remoção de cloretos a partir das cinzas do precipitador da caldeira de recuperação, citam que a concentração de $\mathrm{Cl}^{-}$no licor negro deve permanecer abaixo de $10 \mathrm{~g} / \mathrm{L}$ a fim de evitar corrosão e problemas de entupimentos. Relatam que algumas plantas operam com concentrações de $15 \mathrm{~g} / \mathrm{L}$ devido à flutuação das toras no mar durante o transporte, o que aumenta a concentração de 1 a $3 \%$ para 3 a $5 \%$ de sólidos no licor negro. Em indústrias de ciclo parcial ou totalmente fechado este valor sobe para 5 a $7 \%$.

Com a finalidade de separação do cloreto de sódio no sistema de recuperação de químicos foi projetado um processo contínuo baseado na dissolução seletiva do $\mathrm{NaCl}$ a partir das cinzas do precipitador da caldeira de recuperação. O princípio básico do processo consiste em dissolver preferencialmente o $\mathrm{NaCl}$ em água e subseqüentemente diluir esta solução com o licor percolado reciclado que se encontra saturado com $\mathrm{Na}_{2} \mathrm{SO}_{4}$ e $\mathrm{NaCl}$. A porção insolúvel das cinzas é recuperada por filtração, e o $\mathrm{NaCl}$ dissolvido é removido do sistema (MOY et al., 1974).

Na recuperação, o potássio e os cloretos serão coletados pelo precipitador eletrostático, o manganês pode ser retirado pela filtração do licor verde enquanto que a sílica e o fósforo são separados no forno de cal. Podem se tornar necessárias purgas eventuais de cinzas dos precipitadores eletrostáticos e de lama de cal (EDSTROM, 1994).

Metais como manganês, ferro, cobre, cálcio, etc. podem ser removidos pelo tratamento ácido, e posteriormente por precipitação, em pH adequado, sendo então retirados por filtração. Esta técnica tem a vantagem de eliminar os metais eficientemente evitando a lavagem em contra-corrente e acumulação. Em consequência, tais elementos são enviados para purga constituindo o "dreg" 
(LINDBERG, 1994).

ALBERT (1995) cita que tanto é possível atualmente remodelar tecnologicamente uma fábrica de polpa branqueada em funcionamento para operar sem efluente, reduzindo seu custo operacional, como também projetar, construir e operar uma nova indústria sem efluentes com custo de capital e custos operacionais menores que uma fábrica convencional. Relata que requisitos básicos para isso constituem em utilizar reagentes químicos que sejam recicláveis ou que reajam para formar ar, água, e constituir outros reagentes e, promover a remoção de elementos não-processuais que entram no sistema.

\section{MATERIAL E MÉTODOS}

\section{Descrição e localização da área amostral}

Todas as madeiras amostradas são procedentes de povoamentos florestais de Acacia mearnsii, Eucalyptus dunnii, Eucalyptus globulus, Eucalyptus grandis e Eucalyptus saligna da Riocell S. A., localizados próximos à Guaíba, RS.

A Acacia mearnsii tinha 76 meses de idade quando da coleta. $\mathrm{O}$ espaçamento utilizado no plantio foi de $3 \times 1,33 \mathrm{~m}$.

Os espaçamentos dos povoamentos de todos os eucaliptos eram 3 x $2 \mathrm{~m}$.

O local de coleta para o Eucalyptus dunnii, bem como para Eucalyptus grandis e Eucalyptus saligna foi o Horto Florestal Aldo Sani, localizado em Butiá, RS.

O povoamento de Eucalyptus dunnii foi coletado com 59 meses de idade.

O Eucalyptus globulus, amostrado no Horto Água Boa, tinha 56 meses de idade quando da coleta. O Eucalyptus grandis possuía idade de 59 meses, enquanto o Eucalyptus saligna foi coletado com 57 meses.

As espécies Eucalyptus dunnii, Eucalyptus grandis, e Eucalyptus saligna foram amostradas em talhões experimentais, enquanto o Eucalyptus globulus e a Acacia mearnsii foram amostrados em talhões comerciais.

\section{Coleta de amostras}

A coleta foi realizada dentro de 5 classes de diâmetros cobrindo proporcionalmente os valores observados em inventário florestal. Um total de 15 indivíduos foram amostrados por espécie.

Após o abate das árvores foram retirados discos (em triplicata) na base, 25, 50, 75 e 100\% da altura comercial.

\section{Análises químicas}

Do material coletado, um disco foi usado para suprir material para a análise de elementos 
minerais, um segundo disco, para avaliação de densidade básica, restando um terceiro disco como reserva de material. Para os discos de avaliação de elementos minerais foram tomadas cunhas sem casca com o mesmo ângulo, onde posteriormente parte deles foi transformada em palitos com o auxílio de um facão de titânio (SACON et al, 1994). Parte do material foi reduzido a cinzas, adicionado de $\mathrm{NaOH}$ p.a. As amostras assim preparadas permitiram análises de alumínio, ferro, cobre, manganês, níquel e silício. Esta preparação foi assim executada principalmente para a análise de silício e foi útil aos demais em vista dos baixos valores de emissão dos elementos supra citados, em função de suas baixas concentrações.

O restante dos palitos foi transformado em serragem em moinho tipo Willey. Após, foram tomadas $0,2 \mathrm{~g}$ desta serragem e colocada em tubos para ser submetida a digestão nitroperclórica (adição de ácido perclórico após ação do ácido nítrico). As amostras foram avolumadas e filtradas, sendo analisadas para cálcio, potássio, magnésio e sódio. Foram preparadas soluções padrões dos elementos analisados, a fim de se construir as curvas analíticas para a calibração do aparelho. Todas as amostras foram analisadas em aparelho plasma (Inductive Coupled Plasm - ICP) modelo Optima 3000 marca Perkin Elmer.

Os resultados dos teores de elementos minerais foram expressos em valores base absolutamente seca $(\mathrm{ppm}=\mathrm{mg}$ do elemento mineral por $\mathrm{kg}$ de madeira absolutamente seca).

\section{Características silviculturais e químicas das espécies e povoamentos}

Foram realizadas determinações nas espécies e povoamentos a seguir enumeradas: diâmetro à altura do peito com casca $\left(\mathrm{d}_{\mathrm{cc}}\right)$ e sem casca $\left(\mathrm{d}_{\mathrm{sc}}\right)$; altura total $\left(\mathrm{h}_{\mathrm{t}}\right)$; altura comercial $\left(\mathrm{h}_{\mathrm{c}}\right)$; volume do cerne $\left(\mathrm{v}_{\text {cer }}\right)$, do alburno $\left(\mathrm{v}_{\mathrm{alb}}\right)$, comercial $\left(\mathrm{v}_{\mathrm{c}}\right)$ e total $\left(\mathrm{v}_{\mathrm{t}}\right)$; densidade básica; percentagem de cerne (\%cer) e alburno (\%alb); incrementos médios anual (IMA), em massa de madeira total (IMAMT), comercial (IMAMC) e equivalente em celulose (IMAEQ), quantidades médias de elementos minerais por árvore, quantidades de elementos minerais exportados por hectare, quantidades de elementos minerais previstas a serem exportadas por hectare aos 7 anos e quantidade média de cada elemento mineral que adentra uma fábrica de $1.000 \mathrm{t} / \mathrm{d}$ de celulose não-branqueada.

\section{Determinação das quantidades médias de elementos minerais por árvore}

Foram calculadas pelo produto entre o valor de massa de madeira comercial para a árvore, pela concentração de cada elemento obtida na marcha analítica.

\section{Determinação das quantidades de elementos minerais exportados por hectare}

Foram calculadas através da multiplicação da concentração de cada elemento pela massa de madeira comercial por hectare exportada para o processo de produção.

\section{Determinação das quantidades de elementos minerais previstas a serem exportadas por hectare aos 7 anos}

Foram calculadas pela correção da idade dos povoamentos para 7 anos a partir da quantidade

Ciência Florestal, v.9, n.1, 1999 
de elementos exportados por hectare, tomando por base o incremento médio anual em madeira comercial, admitindo-se que os teores dos elementos minerais se manterão iguais.

\section{Determinação da quantidade média de cada elemento mineral que adentra uma fábrica de 1000 t/d de celulose não-branqueada}

A carga de elementos minerais que adentra a fábrica é o produto da concentração destes elementos que se encontram na madeira, pela quantidade de madeira necessária para a fabricação de uma determinada quantidade de celulose $(1.000 \mathrm{t} / \mathrm{d})$. Adotou-se, como rendimento do processo de conversão da madeira em celulose, o valor de $50 \%$.

\section{RESULTADOS E DISCUSSÃO}

\section{Quantificação dos elementos minerais}

A tabela 2 relata os valores encontrados nas análises dos elementos minerais. Os mesmos estão em base madeira absolutamente seca, o que facilita a conversão para $\mathrm{kg} / \mathrm{ha} \mathrm{e} \mathrm{kg} / \mathrm{t} \mathrm{de} \mathrm{madeira.}$

TABELA 2: Teores médios de elementos encontrados por espécie (expressos em ppm).

\begin{tabular}{c|c|c|c|c|c}
\hline Elementos & $\begin{array}{c}\text { Acacia } \\
\text { mearnsii }\end{array}$ & $\begin{array}{c}\text { Eucalyptus } \\
\text { dunnii }\end{array}$ & $\begin{array}{c}\text { Eucalyptus } \\
\text { globulus }\end{array}$ & $\begin{array}{c}\text { Eucalyptus } \\
\text { grandis }\end{array}$ & $\begin{array}{c}\text { Eucalyptus } \\
\text { saligna }\end{array}$ \\
\hline $\mathrm{K}$ & 860 & 1020 & 1363 & 823 & 704 \\
$\mathrm{Ca}$ & 849 & 955 & 652 & 541 & 519 \\
$\mathrm{Mg}$ & 261 & 606 & 243 & 182 & 192 \\
$\mathrm{Na}$ & 361 & 264 & 112 & 243 & 458 \\
$\mathrm{Al}$ & 70 & 96 & 36 & 53 & 154 \\
$\mathrm{Mn}$ & 8,9 & 63,0 & 45,1 & 16,6 & 15,0 \\
$\mathrm{Si}$ & 7,2 & 12,3 & 14,5 & 13,0 & 25,0 \\
$\mathrm{Fe}$ & 2,27 & 3,56 & 2,61 & 3,52 & 5,33 \\
$\mathrm{Cu}$ & 1,31 & 1,07 & 1,73 & 1,11 & 1,43 \\
$\mathrm{Ni}$ & 0,06 & 0,23 & 0,38 & 0,12 & 0,27 \\
\hline
\end{tabular}

\section{Características silviculturais das árvores}

A Tabela 3 mostra as características silviculturais médias para cada conjunto de 15 árvores amostradas para cada espécie e a Tabela 4 as convenções utilizadas no trabalho.

$\mathrm{Na}$ Tabela 3 são mostradas as médias para as árvores das espécies estudadas. Enquanto Eucalyptus grandis e Eucalyptus saligna apresentaram árvores volumosas, mas de madeira menos densa, Eucalyptus globulus, Eucalyptus dunnii e Acacia mearnsii mostraram árvores menos volumosas, mas de maior densidade básica. Entretanto, apesar das menores densidades básicas, as árvores médias de Eucalyptus grandis e Eucalyptus saligna foram também as mais pesadas. 
TABELA 3: Características silviculturais médias das árvores.

\begin{tabular}{|c|c|c|c|c|c|c|}
\hline Variáveis & Unidade & $\begin{array}{c}\text { Acacia } \\
\text { mearnsii }\end{array}$ & $\begin{array}{c}\text { Eucalyptus } \\
\text { dunnii }\end{array}$ & $\begin{array}{l}\text { Eucalyptus } \\
\text { globulus }\end{array}$ & $\begin{array}{c}\text { Eucalyptus } \\
\text { grandis }\end{array}$ & $\begin{array}{c}\text { Eucalyptus } \\
\text { saligna }\end{array}$ \\
\hline $\mathrm{d}_{\mathrm{cc}}$ & $\mathrm{cm}$ & 14,6 & 16,7 & 15,0 & 18,1 & 17,1 \\
\hline $\mathrm{d}_{\mathrm{sc}}$ & $\mathrm{cm}$ & 14,0 & 14,9 & 13,9 & 16,7 & 15,9 \\
\hline$h_{t}$ & $\mathrm{~m}$ & 17,48 & 22,48 & 14,81 & 24,01 & 22,94 \\
\hline $\mathrm{h}_{\mathrm{c}}$ & $\mathrm{m}$ & 12,45 & 16,85 & 10,30 & 18,88 & 17,78 \\
\hline$f_{c}$ & - & 0,697 & 0,629 & 0,603 & 0,609 & 0,613 \\
\hline $\mathrm{f}_{\mathrm{t}}$ & - & 0,514 & 0,478 & 0,430 & 0,478 & 0,479 \\
\hline $\mathrm{v}_{\mathrm{C} \text { arv }}$ & $\mathrm{m}^{3}$ & 0,145 & 0,202 & 0,099 & 0,282 & 0,239 \\
\hline $\mathrm{V}_{\mathrm{T} \text { arv }}$ & $\mathrm{m}^{3}$ & 0,165 & 0,258 & 0,120 & 0,335 & 0,281 \\
\hline $\mathrm{v}_{\text {cer }}$ & $\mathrm{m}^{3}$ & 0,070 & 0,067 & 0,014 & 0,083 & 0,061 \\
\hline$V_{\text {alb }}$ & $\mathrm{m}^{3}$ & 0,075 & 0,136 & 0,085 & 0,199 & 0,178 \\
\hline $\mathrm{v}_{\text {cas }}$ & $\mathrm{m}^{3}$ & 0,013 & 0,049 & 0,015 & 0,046 & 0,036 \\
\hline $\mathrm{db}_{\text {cer }}$ & $\mathrm{g} / \mathrm{cm}^{3}$ & 0,519 & 0,449 & 0,484 & 0,424 & 0,400 \\
\hline $\mathrm{db}_{\mathrm{alb}}$ & $\mathrm{g} / \mathrm{cm}^{3}$ & 0,617 & 0,490 & 0,516 & 0,439 & 0,461 \\
\hline $\mathrm{db}_{\mathrm{arv}}$ & $\mathrm{g} / \mathrm{cm}^{3}$ & 0,566 & 0,477 & 0,511 & 0,438 & 0,453 \\
\hline$M_{\text {cer }}$ & $\mathrm{kg}$ & 36,901 & 30,619 & 6,775 & 35,639 & 25,928 \\
\hline $\mathrm{M}_{\mathrm{alb}}$ & $\mathrm{kg}$ & 45,947 & 68,155 & 44,206 & 88,433 & 81,895 \\
\hline $\mathbf{M}_{\text {mad }}$ & $\mathrm{kg}$ & 82,847 & 98,774 & 50,981 & 124,072 & 107,824 \\
\hline cer/alb (M) & - & 0,8031 & 0,4493 & 0,1532 & 0,4030 & 0,3166 \\
\hline cer/alb(v) & - & 0,9389 & 0,4896 & 0,1646 & 0,4164 & 0,3441 \\
\hline \%cerne $\left(\mathrm{v}_{\mathrm{C}}\right)$ & $(\mathrm{kg} / \mathrm{kg})$ & 44,5 & 31,0 & 13,3 & 28,7 & 24,0 \\
\hline$\%$ alburno $\left(\mathrm{v}_{\mathrm{C}}\right)$ & $(\mathrm{kg} / \mathrm{kg})$ & 55,5 & 69,0 & 86,7 & 71,3 & 76,0 \\
\hline$\% \operatorname{casca}\left(\mathrm{v}_{\mathrm{T}}\right)$ & $\left(\mathrm{m}^{3} / \mathrm{m}^{3}\right)$ & 8,2 & 19,1 & 12,9 & 13,7 & 12,8 \\
\hline$\%$ cinzas & - & 0,57 & 0,71 & 0,65 & 0,38 & 0,41 \\
\hline
\end{tabular}

A quantidade de cerne foi mais significativa em Acacia mearnsii, enquanto que as árvores que tinham mais casca foram as de Eucalyptus dunnii.

Em termos de teores de cinzas, ou seja, dos minerais totais presentes na madeira em suas mais diferentes formas, as espécies Eucalyptus dunnii e Eucalyptus globulus foram as mais expressivas. Essa característica é indesejável, pois pode ser um fator negativo no uso dessas madeiras em fábricas de celulose de ciclo de água muito fechado, com altas taxas de recirculações e baixos volumes de efluentes. 
TABELA 4: Convenções utilizadas no trabalho.

\begin{tabular}{cll}
\hline Convenção & \multicolumn{1}{c}{ Significado } \\
\hline $\mathrm{d}_{\mathrm{cc}}$ & diâmetro à altura do peito com casca $(\mathrm{cm})$ \\
$\mathrm{d}_{\mathrm{sc}}$ & diâmetro à altura do peito sem casca $(\mathrm{cm})$ \\
$\mathrm{h}_{\mathrm{t}}$ & altura total $(\mathrm{m})$ \\
$\mathrm{h}_{\mathrm{c}}$ & altura comercial $(\mathrm{m})$ \\
$\mathrm{v}_{\mathrm{x}}$ & volume, sendo $\mathrm{x}=$ cerne, alburno ou madeira $\left(\mathrm{m}^{3}\right)$ \\
$\mathrm{v}_{\mathrm{T}}$ & volume total $\left(\mathrm{m}^{3}\right)$ \\
$\mathrm{v}_{\mathrm{C}}$ & volume comercial $\left(\mathrm{m}^{3}\right)$ \\
$\mathrm{f}_{\mathrm{t}}$ & fator de forma total $\left(\mathrm{h}_{\mathrm{t}}, \mathrm{d}_{\mathrm{cc}}\right)$ \\
$\mathrm{f}_{\mathrm{c}}$ & fator de forma comercial $\left(\mathrm{hc}, \mathrm{d}_{\mathrm{sc}}\right)$ \\
$\mathrm{M}$ & massa $(\mathrm{kg} ; \mathrm{t})$ \\
$\mathrm{db}$ & densidade básica $\left(\mathrm{t} / \mathrm{m}^{3}=\mathrm{g} / \mathrm{cm}^{3}\right)$ \\
$\mathrm{mad}$ & madeira $\left(\mathrm{m}^{3} ; \mathrm{kg} ; \mathrm{t}\right)$ \\
$\mathrm{cer}$ & cerne $\left(\mathrm{m}^{3} ; \mathrm{kg} ; \mathrm{t}\right)$ \\
$\mathrm{alb}$ & alburno $\left(\mathrm{m}^{3} ; \mathrm{kg} ; \mathrm{t}\right)$ \\
$\mathrm{arv}$ & árvore \\
$\mathrm{IMA}$ & incremento médio anual $\left(\mathrm{m}^{3} / \mathrm{ha} \cdot \mathrm{a}\right)$ \\
$\mathrm{IMAT}$ & IMA em volume total $\left(\mathrm{m}^{3} / \mathrm{ha} . \mathrm{a}\right)$ \\
$\mathrm{IMAC}$ & IMA em volume comercial $\left(\mathrm{m}^{3} / \mathrm{ha} . \mathrm{a}\right)$ \\
$\mathrm{IMAMT}$ & IMA em massa de madeira total $(\mathrm{t} / \mathrm{ha} . \mathrm{a})$ \\
$\mathrm{IMAMC}$ & IMA em massa de madeira comercial $(\mathrm{t} / \mathrm{ha} . \mathrm{a})$ \\
$\mathrm{IMAEQ}$ & IMA em massa equivalente de celulose $(\mathrm{t} / \mathrm{ha} . \mathrm{a})$ \\
EDTA & ácido etilenodiaminotetracético \\
DTPA & ácido dietilenotriaminopentacético \\
ECF & Elemental Chlorine Free $($ ausência de cloro elementar $)$ \\
TCF & Totally Chlorine Free $($ ausência total de cloro $)$ \\
TEF & Totally Effluent Free $($ ausência de efluentes $)$ \\
\hline
\end{tabular}

Fonte: FINGER \& SCHNEIDER (1988).

\section{Características silviculturais dos povoamentos}

A Tabela 5 apresenta as características silviculturais médias de povoamentos florestais, estimadas a partir dos dados médios das árvores e das taxas de sobrevivência e dos espaçamentos de plantio.

De acordo com a Tabela 5, em termos de produção de massa (IMAMC), de volume $\left(\mathrm{v}_{\mathrm{C}}, \mathrm{v}_{\mathrm{T}}\right)$ e de massa equivalente em celulose (IMAEQ), as espécies Eucalyptus grandis e Eucalyptus saligna $\left(34,554\right.$ e $30,621 \mathrm{t} / \mathrm{ha*}$ a; 387,755 e $321,213 \mathrm{~m}^{3} / \mathrm{ha} ; 460,06$ e $378,381 \mathrm{~m}^{3} / \mathrm{ha} ; 17,277$ e $15,31 \mathrm{t} / \mathrm{ha*}$, respectivamente) confirmaram suas excelentes performances, justificando com facilidade as razões que as levam a serem as preferidas para uso industrial na fabricação de celulose no sul do Brasil. Entretanto, os dados dos povoamentos mostram uma certa heterogeneidade em vários aspectos. Primeiramente, deve-se ter em conta que os povoamentos de Acacia mearnsii e Eucalyptus globulus 
eram comerciais enquanto os demais eram experimentais. Em segundo lugar que, embora as idades dos povoamentos de eucaliptos eram relativamente semelhantes, Acacia mearnsii era diferente. Outro dado que traz uma separação grande entre as espécies é a taxa de sobrevivência, baixa em Acacia mearnsii e Eucalyptus globulus.

TABELA 5: Características silviculturais dos povoamentos florestais.

\begin{tabular}{l|c|c|c|c|c}
\hline \multicolumn{1}{c|}{ Características } & $\begin{array}{c}\text { Acacia } \\
\text { mearnsii }\end{array}$ & $\begin{array}{c}\text { Eucalyptus } \\
\text { dunnii }\end{array}$ & $\begin{array}{c}\text { Eucalyptus } \\
\text { globulus }\end{array}$ & $\begin{array}{c}\text { Eucalyptus } \\
\text { grandis }\end{array}$ & $\begin{array}{c}\text { Eucalyptus } \\
\text { saligna }\end{array}$ \\
\hline Sobrevivência (\%) & 61,3 & 79,0 & 60,7 & 82,6 & 80,7 \\
Idade (meses) & 76 & 59 & 56 & 59 & 57 \\
IMAT $^{1}$ & 40,048 & 54,667 & 25,905 & 93,572 & 79,659 \\
IMAC $^{1}$ & 35,162 & 42,800 & 21,469 & 78,865 & 67,624 \\
IMAMC $^{2}$ & 19,886 & 20,397 & 10,979 & 34,554 & 30,621 \\
IMAEQ $^{2}$ & 9,943 & 10,198 & 5,489 & 17,277 & 15,310 \\
v $_{\mathrm{C}}{ }^{3}$ & 22,690 & 210,433 & 100,189 & 387,755 & 321,213 \\
$\mathrm{v}_{\mathrm{T}}{ }^{3}$ & 253,640 & 268,781 & 120,891 & 460,060 & 378,381 \\
\hline
\end{tabular}

${ }^{1} \mathrm{~m}^{3} /$ ha.a; ${ }^{2} \mathrm{t} / \mathrm{ha} . \mathrm{a} ;{ }^{3} \mathrm{~m}^{3} /$ ha, na amostragem.

\section{Quantidades de elementos exportados via madeira}

As Tabelas 6, 7 e 8 apresentam as quantidades de elementos minerais contidos na estrutura da madeira. A Tabela 6 mostra a quantidade em gramas de cada elemento mineral apresentado em

TABELA 6: Quantidades médias de elementos minerais presentes na madeira comercial por árvore para as espécies estudadas (g/árvore).

\begin{tabular}{c|c|c|c|c|c}
\hline Elementos & $\begin{array}{c}\text { Acacia } \\
\text { mearnsii }\end{array}$ & $\begin{array}{c}\text { Eucalyptus } \\
\text { dunnii }\end{array}$ & $\begin{array}{c}\text { Eucalyptus } \\
\text { globulus }\end{array}$ & $\begin{array}{c}\text { Eucalyptus } \\
\text { grandis }\end{array}$ & $\begin{array}{c}\text { Eucalyptus } \\
\text { saligna }\end{array}$ \\
\hline $\mathrm{K}$ & 71,5 & 98,1 & 68,9 & 101,7 & 76,0 \\
$\mathrm{Ca}$ & 70,6 & 91,8 & 33,0 & 66,8 & 56,0 \\
$\mathrm{Mg}$ & 21,7 & 58,3 & 12,3 & 22,5 & 20,7 \\
$\mathrm{Na}$ & 29,98 & 25,38 & 5,64 & 29,96 & 49,37 \\
$\mathrm{Al}$ & 5,86 & 9,22 & 1,80 & 6,54 & 16,65 \\
$\mathrm{Mn}$ & 0,744 & 6,056 & 2,282 & 2,046 & 1,614 \\
$\mathrm{Si}$ & 0,597 & 1,183 & 0,733 & 1,609 & 2,693 \\
$\mathrm{Fe}$ & 0,189 & 0,342 & 0,132 & 0,434 & 0,575 \\
$\mathrm{Cu}$ & 0,109 & 0,103 & 0,087 & 0,137 & 0,155 \\
$\mathrm{Ni}$ & 0,005 & 0,022 & 0,019 & 0,015 & 0,030 \\
\hline Total & 201,305 & 290,559 & 124,877 & 231,709 & 223,771 \\
Total cinzas & 474,109 & 587,305 & 539,719 & 318,731 & 337,549 \\
$\%$ elementos & 42,460 & 49,473 & 23,137 & 72,697 & 66,293 \\
\hline
\end{tabular}

${ }^{1}$ porcentagem dos elementos minerais estudados nas cinzas. 
TABELA 7: Quantidades de elementos minerais exportados com a madeira comercial (kg/ha).

\begin{tabular}{c|c|c|c|c|c}
\hline Elementos & $\begin{array}{c}\text { Acacia } \\
\text { mearnsii }\end{array}$ & $\begin{array}{c}\text { Eucalyptus } \\
\text { dunnii }\end{array}$ & $\begin{array}{c}\text { Eucalyptus } \\
\text { globulus }\end{array}$ & $\begin{array}{c}\text { Eucalyptus } \\
\text { grandis }\end{array}$ & $\begin{array}{c}\text { Eucalyptus } \\
\text { saligna }\end{array}$ \\
\hline $\mathrm{K}$ & 109,9 & 102,1 & 69,8 & 139,8 & 102,2 \\
$\mathrm{Ca}$ & 108,5 & 95,5 & 33,4 & 91,8 & 75,3 \\
$\mathrm{Mg}$ & 33,3 & 60,6 & 12,4 & 30,9 & 27,8 \\
$\mathrm{Na}$ & 46,1 & 26,4 & 5,7 & 41,2 & 66,4 \\
$\mathrm{Al}$ & 8,999 & 9,593 & 1,821 & 8,990 & 22,399 \\
$\mathrm{Mn}$ & 1,143 & 6,298 & 2,311 & 2,813 & 2,171 \\
$\mathrm{Si}$ & 0,917 & 1,230 & 0,743 & 2,212 & 3,623 \\
$\mathrm{Fe}$ & 0,290 & 0,356 & 0,134 & 0,597 & 0,774 \\
$\mathrm{Cu}$ & 0,168 & 0,107 & 0,088 & 0,189 & 0,208 \\
$\mathrm{Ni}$ & 0,008 & 0,023 & 0,019 & 0,021 & 0,040 \\
\hline
\end{tabular}

média por árvore (volume comercial). As Tabelas 7 e 8 apresentam as quantidades removidas por hectare, na idade da amostragem e a previsão quando da colheita aos 7 anos, respectivamente.

$\mathrm{Na}$ Tabela 8, valores em $\mathrm{kg} / \mathrm{ha}$ dos elementos que serão exportados do solo pela madeira quando da ocasião do corte, que é projetado para os 7 anos de idade, estão inclusos apenas os elementos estudados que realmente adentrarão à fábrica. As diferenças entre espécies não são apenas em função dos teores de elementos nas madeiras mas também da produção volumétrica e em peso das espécies estudadas. Espécies de baixa produtividade florestal tendem a exportar menos por hectare, já que a quantidade de madeira produzida entra nos cálculos de exportação.

TABELA 8. Quantidades de elementos exportados por hectare, km/ha (previsão para 7 anos).

\begin{tabular}{c|c|c|c|c|c}
\hline Elementos & $\begin{array}{c}\text { Acacia } \\
\text { mearnsii }\end{array}$ & $\begin{array}{c}\text { Eucalyptus } \\
\text { dunnii }\end{array}$ & $\begin{array}{c}\text { Eucalyptus } \\
\text { globulus }\end{array}$ & $\begin{array}{c}\text { Eucalyptus } \\
\text { grandis }\end{array}$ & $\begin{array}{c}\text { Eucalyptus } \\
\text { saligna }\end{array}$ \\
\hline $\mathrm{K}$ & 121,5 & 145,3 & 104,5 & 199,0 & 150,6 \\
$\mathrm{Ca}$ & 119,8 & 136,0 & 50,0 & 130,8 & 111,0 \\
$\mathrm{Mg}$ & 36,829 & 86,258 & 18,632 & 44,060 & 41,010 \\
$\mathrm{Na}$ & 50,901 & 37,581 & 8,556 & 58,641 & 97,851 \\
$\mathrm{Al}$ & 9,941 & 13,657 & 2,728 & 12,799 & 33,009 \\
$\mathrm{Mn}$ & 1,262 & 8,967 & 3,461 & 4,005 & 3,199 \\
$\mathrm{Si}$ & 1,013 & 1,751 & 1,112 & 3,149 & 5,339 \\
$\mathrm{Fe}$ & 0,321 & 0,506 & 0,200 & 0,850 & 1,140 \\
$\mathrm{Cu}$ & 0,185 & 0,152 & 0,132 & 0,268 & 0,306 \\
$\mathrm{Ni}$ & 0,008 & 0,033 & 0,029 & 0,030 & 0,059 \\
\hline $\mathrm{Soma}$ & 341,76 & 430,205 & 189,35 & 453,602 & 443,513 \\
\hline
\end{tabular}

Pode-se verificar que a soma de elementos exportados por hectare foi menor em Eucalyptus globulus que as demais espécies. Entretanto, se for comparado o valor de produção de madeira para esta mesma espécie, ou seja 120,891 $\mathrm{m}^{3} /$ ha, pode ser verificado que este valor também é pequeno. 


\section{Quantidades médias de elementos minerais que ingressam na fabricação de celulose via madeira}

As quantidades de elementos minerais que ingressam na fábrica de celulose foram estimadas com base nos teores destes elementos e na quantidade de madeira requerida.

Considerou-se para isso uma unidade fabril produzindo $1.000 \mathrm{t} / \mathrm{d}$ de celulose com um rendimento de $50 \%$ na conversão da madeira para celulose, independente da espécie. Dessa forma, a fábrica necessita de $2.000 \mathrm{t} / \mathrm{d}$ de madeira. Com base nos teores encontrados na madeira $\mathrm{e}$ apresentados na Tabela 2, foi possível construir a Tabela 9.

TABELA 9: Quantidades médias de elementos que ingressam pela madeira em um dia de produção de uma fábrica de $1.000 \mathrm{t} / \mathrm{d}$ de celulose.

\begin{tabular}{c|c|c|c|c|c|c}
\hline Elementos & Unidade & $\begin{array}{c}\text { Acacia } \\
\text { mearnsii }\end{array}$ & $\begin{array}{c}\text { Eucalyptus } \\
\text { dunnii }\end{array}$ & $\begin{array}{c}\text { Eucalyptus } \\
\text { globulus }\end{array}$ & $\begin{array}{c}\text { Eucalyptus } \\
\text { grandis }\end{array}$ & $\begin{array}{c}\text { Eucalyptus } \\
\text { saligna }\end{array}$ \\
\hline $\mathrm{K}$ & $\mathrm{t} / \mathrm{d}$ & 1,720 & 2,041 & 2,725 & 1,647 & 1,408 \\
$\mathrm{Ca}$ & $\mathrm{t} / \mathrm{d}$ & 1,698 & 1,910 & 1,305 & 1,082 & 1,039 \\
$\mathrm{Mg}$ & $\mathrm{t} / \mathrm{d}$ & 0,522 & 1,211 & 0,486 & 0,365 & 0,384 \\
$\mathrm{Na}$ & $\mathrm{t} / \mathrm{d}$ & 0,721 & 0,528 & 0,223 & 0,485 & 0,915 \\
$\mathrm{Al}$ & $\mathrm{kg} / \mathrm{d}$ & 140,819 & 191,805 & 71,130 & 105,887 & 308,754 \\
$\mathrm{Mn}$ & $\mathrm{kg} / \mathrm{d}$ & 17,882 & 125,931 & 90,262 & 33,134 & 29,922 \\
$\mathrm{Si}$ & $\mathrm{kg} / \mathrm{d}$ & 14,347 & 24,598 & 29,001 & 26,054 & 49,937 \\
$\mathrm{Fe}$ & $\mathrm{kg} / \mathrm{d}$ & 4,544 & 7,113 & 5,215 & 7,033 & 10,668 \\
$\mathrm{Cu}$ & $\mathrm{kg} / \mathrm{d}$ & 2,626 & 2,136 & 3,451 & 2,221 & 2,866 \\
$\mathrm{Ni}$ & $\mathrm{kg} / \mathrm{d}$ & 0,118 & 0,461 & 0,752 & 0,249 & 0,548 \\
\hline $\mathrm{Total}$ & $\mathrm{t} / \mathrm{d}$ & 4,841 & 6,042 & 4,938 & 3,753 & 4,149 \\
\hline
\end{tabular}

Conforme a Tabela 9, a espécie que mais contribui com o conjunto dos elementos que entram na indústria via madeira é o Eucalyptus dunnii, seguido por Eucalyptus globulus, Acacia mearnsii, Eucalyptus saligna e Eucalyptus grandis. Em termos da massa dos elementos minerais, a carga do Eucalyptus dunnii foi 6,042 t por dia. Os valores decresceram em Eucalyptus globulus (4,938 t), Acacia mearnsii (4,841 t), Eucalyptus saligna (4,149 t) e Eucalyptus grandis (3,753 t).

A produção de celulose com Eucalyptus grandis, deste ponto de vista seria a mais sensata, uma vez que poderia proporcionar menores problemas na fábrica. Ao contrário, o uso de Eucalyptus dunnii significa um acréscimo de cerca de $60 \%$ na carga de elementos ao processo. É importante ressaltar que não é apenas a quantidade de elementos que precisa ser considerada, mas também qual elemento. Conforme o projeto do processo e a tecnologia empregada, um ou outro elemento pode adquirir relevância nos problemas que venha a causar.

Por outro lado, ao se analisar o teor de cinzas nas madeiras, para a mesma fábrica de 1.000 $\mathrm{t} / \mathrm{d}$, as quantidades dessas que ingressam na mesma, por dia, variam entre 8 a $14 \mathrm{~kg}$ cinzas $/ \mathrm{t}$ de celulose. Isso se deve ao fato que, ao se determinar cinzas, avaliam-se outros elementos minerais, além dos pesquisados e a análise inclui também ânions como óxidos, carbonatos, sulfatos, etc. 


\section{CONCLUSÕES E RECOMENDAÇÕES}

Da análise dos resultados foi possível obter as seguintes conclusões:

a) é importante a quantidade de elementos minerais presentes nas madeiras e sua variabilidade, o que significa remoções das áreas florestais durante a colheita da madeira, bem como ingressos desses minerais nas fábricas de celulose junto com a madeira, com possibilidades de causar problemas industriais;

b) os elementos minerais cujas presenças nas madeiras são mais abundantes são: potássio, cálcio, magnésio, sódio, alumínio, manganês e silício;

c) elementos em menor quantidade, mas importantes o suficiente para causarem problemas em fábricas de celulose de ciclo fechado foram: ferro, cobre e níquel;

d) espécies de alta produtividade florestal como Eucalyptus grandis e Eucalyptus saligna ratificaram por essa característica e por um conteúdo mineral menor a importância dessas espécies para o fabricante de celulose no Brasil;

e) espécies como Eucalyptus dunnii e Eucalyptus globulus, ao mesmo tempo que mostraram menores incrementos florestais, apresentaram madeiras mais ricas em cinzas e elementos minerais. Dessa maneira, são espécies onde são exigidos maiores cuidados florestais (maiores exportadores de nutrientes) e industriais (maiores cargas de minerais ao processo);

f) a madeira de Acacia mearnsii apresentou vantagens quanto à menor presença de ferro, manganês e níquel na madeira, sendo mais indicada para branqueamento TCF, conforme já observado em operação industrial;

g) as quantidades de $\mathrm{Si}$ encontradas foram baixas, sugerindo que os altos teores de sílica observados na polpação parecem não ser originários da madeira, mas de contaminação das toras com terra durante o manuseio das mesmas, operações que também introduzem outros elementos minerais. Desta forma, as empresas de celulose devem avaliar melhor a operação das unidades de lavagem de toras, visando conhecer sua performance e otimizá-la;

h) a espécie que menos introduz elementos minerais em $\mathrm{kg} / \mathrm{t}$ de celulose para o processo industrial é Eucalyptus grandis $(3,753)$, seguido de Eucalyptus saligna $(4,149)$;

i) a espécie que introduz maiores teores de elementos minerais em $\mathrm{kg} / \mathrm{t}$ de celulose ao processo é Eucalyptus dunnii (6,042), seguida de Eucalyptus globulus (4,938);

Diante do exposto recomenda-se que tanto as áreas florestais como industriais, bem como os fabricantes de equipamentos e geradores de tecnologia para o setor de celulose e papel, coloquem esforços e mais atenção na problemática de exportação de elementos minerais das florestas e conseqüente ingresso dos mesmos nos processos de fabricação como elementos indesejáveis. 


\section{REFERÊNCIAS BIBLIOGRÁFICAS}

ALBERT, R. J. Technical and economic feasibility of the effluent-free bleached kraft pulp mill. In: INTERNATIONAL NON-CHLORINE BLEACHING CONFERENCE, 1993 [s.1.], 1993. 23 p. inclui material em anexo

ALBERT, R. J. Worldwide status of effluent-free technology for bleached kraft pulp production. In: INTERNATIONAL NON-CHLORINE BLEACHING CONFERENCE, 1995 [s.l.], 1995. 37 p. inclui material em anexo

ALBERT, R. J. Current status of TEF bleached kraft pulp mills and future trends. In: INTERNATIONAL NON-CHLORINE BLEACHING CONFERENCE, 1996 [s.l.], 1996. 38 p. inclui material em anexo

BRYANT, P. S., EDWARDS, L. L. Monitoring and control of transition element in kraft mill fiberlines. [S.1.: s.n.],1994. 23p. inclui material em anexo.

DEVENYNS, J.; BALlADA, F. D.; TROUGHTON, N.; PLUMET, L.; Control de metales en un blanqueo TCF. El Papel, n. 48, p. 24-33, abril, 1995.

EDSTROM, S. The 'closed' chemical pulp mill: myth or reality? Papermaker, p. 24-27, September, 1994.

FINGER, C. A. G.; SCHNEIDER, P. R. Padronização de símbolos florestais. Série Técnica, n.5, UFSM/CEPEF/FATEC, Santa Maria, 1988, 11 p.

FOELKEL, C., ASSIS, T.F. New pulping technology and Eucalyptus wood: the role of soil fertility, plant nutrition and wood ion content. In: EUCALYPTUS PLANTATIONS: IMPROVING FIBRE YELD AND QUALITY, 1995. Hobart. Proceedings... Sandy Bay: Cooperative Research Centre for Temperate Hardwood Forestry, 1995. 488 p. p.10-13.

GLEADOW, P., HASTINGS, C. S., JOHNSON, T. et al. A survey of research and development activity in bleached kraft mill closed cycle. In: INTERNATIONAL NON CHLORINE BLEACHING CONFERENCE, 1993. [S.1.] 1993. 9 p.

GRACE, T. M., SACHS, D. G.,GRADY, H. J. Determination of the inorganic composition of alcaline black liquors. Tappi. v. 60, n. 4, p. 122-125, 1977.

HOBBS, G. C., ABBOT, J. The effect of aluminum on the two-stage peroxide bleaching of Eucalyptus regnans stone groundwood pulp. Journal of Pulp and Paper Science. v. 20, n. 2, p. j50-j54. 1994.

LINDBERG, H. Strategies for metal removal control in closed cycle mills. In: INTERNATIONAL PULP BLEACHING CONFERENCE, 1994. Vancouver, 1994. p. 293-302.

MOUDGIL, B. M., PRAKASH, T. S. Application of aqueous-aqueous separation technique for chloride removal from precipitator salt cake. [S.1.: s.n., 19_] 23p.

MOY, W. A., JOYCE, P., STYAM, G. E. Removal of sodium chloride from kraft recovery systems. Pulp and Paper Magazine of Canada, v. 75, n. 4, p.88-90, 1974. 
PERKIN ELMER. Optima 3000 ICP Emission Spectrometer. Catálogo. Norwalk, 1992.

SACON, V. M.; DIAS, C. A.; FOELKEL, C.; ASSIS, T. F. Metais em madeira X preparação amostral. [Guaíba] : Riocell, 1994. (Relatório Técnico, 1091)

STRÖMQVIST, D. New technology development for the closed cycle bleach plant. In: SECOND GLOBAL CONFERENCE ON PAPER \& THE ENVIRONMENT, 1994, Frankfurt. Anais... Frankfurt. 1994.

TRAN, H. N., BARHAM, D., REEVE, D. W. Chloride and potassium in the kraft chemical recovery cycle, Pulp and Paper Canada, v.91, n.5, p.55-62, 1990.

VENTURA, J. W., TURQUETTI, A. A. Balanço de cloretos e potássio no sistema de licor. [Guaíba] : Riocell, 1994. n. p. (Relatório Técnico, 718). 